\section{Le rôle du neuropeptide GnRH dans la cascade gonadotrope enfin validé dans l'espèce humaine}

Jérôme Bouligand, Anne Guiochon-Mantel, Jacques Young
L'axe hypothalamo-hypophysaire et le contrôle des fonctions gonadiques Le rôle fondamental de l'hypothalamus dans la commande des fonctions hypophysaires, tout particulièrement celles qui contrôlent les fonctions gonadiques et le développement pubertaire, a été évoqué depuis des décennies [1]. C'est bien plus tard, en 1973, grâce aux travaux des deux prix Nobel Andrew Schally et Roger Guillemin, que le neuropeptide $\mathrm{GnRH}$ (gonadotropin releasing hormone) qui contrôle la sécrétion des hormones FSH (folliculo stimulating hormone) et LH (luteinizing hormone) par les cellules gonadotropes hypophysaires a été isolé et sa séquence peptidique, parfaitement conservée chez les mammifères, établie [2]. Quatre années plus tard, en 1977, un modèle animal naturel déficitaire en GnRH hypothalamique était décrit; les animaux présentaient un hypogonadisme hypogonadotrophique isolé (avec une baisse concomitante des gonadotrophines hypophysaires et des stéroïdes sexuels), d'où son appellation de souris hpg [3]. Mais le produit du gène incriminé n'était pas identifié. Parallèlement, le gène codant pour le précurseur du décapeptide mature GnRH était identifié et caractérisé par le groupe de Seeburg [4]. Depuis, deux gènes de la GnRH ont été identifiés dans plusieurs espèces et chez l'homme [5]. C'est le gène codant pour la GnRH de type 1 (GNRHI) qui est impliqué dans la cascade gonadotrope. Le GNRHI, localisé chez l'homme sur le bras court du chromosome 8 en 8p21-p11.2, contient 4 exons séparés par 3 introns (Figure 1). Le premier exon code pour une partie 5' non traduite, le second pour le peptide signal du GnRH, le décapeptide et les 11 premiers acides aminés du peptide associé au GnRH (GnRH associated peptide, GAP). Les troisième et quatrième exons codent pour les acides aminés restants du GAP et le quatrième exon contient aussi la partie 3' non traduite. La séquence décapeptidique de la GnRH (Figure 2), identique chez tous les mammifères, et le rôle des acides aminés la composant sont, répétons-le, extrêmement conservés à travers l'évolution avec une fonction précise des différentes régions du décapeptide [5].

\section{Le lien entre la souris hpg (hypogonadisme hypogonadotrophique) et le gène GNRH}

C'est seulement en 1986 que Mason et al. font la relation entre la souris hpg et le gène codant pour la GnRH. Ce groupe décrit une délétion de $33,5 \mathrm{~kb}$ incluant le troisième et le quatrième exons de gnrhl chez cette souris [6]. Celle-ci est depuis les années 1980 un paradigme naturel de déficit gonadotrope dû à une carence en GnRH largement utilisé par la communauté scientifique pour explorer les fonctions hypothalamique, hypophysaire et gonadique des mammifères. Chez ces souris, les gonadotrophines hypophysaires sont sécrétées en réponse à la GnRH exogène [7], ce qui montre une atteinte exclusivement hypothalamique. Dans ce modèle, le
J. Bouligand, A. Guiochon-Mantel :

Université Paris-Sud 11 et Inserm U693, Faculté de médecine Paris Sud.

Service de génétique moléculaire, pharmacogénétique et hormonologie, Centre hospitalier universitaire de Bicêtre, Assistance Publique-Hôpitaux de Paris, France.

J. Young : Université Paris-Sud 11 et Inserm U693,

Faculté de médecine Paris Sud.

Service d'endocrinologie

et des maladies de la reproduction.

Centre Hospitalier Universitaire de Bicêtre, Assistance Publique-Hôpitaux de Paris,

78, rue du Général Leclerc,

94275 Le Kremlin Bicêtre, France.

jacques.young@bct.aphp.fr

gnrhl était transcrit mais aucune immunoréactivité de la GnRH n'était détectée dans I'hypothalamus. Ainsi, bien que les neurones à GnRH soient normalement présents et correctement localisés au niveau de l'hypothalamus, il n'y avait pas, chez les souris hpg, de sécrétion du décapeptide mature $[7,8]$. Le lien entre le génotype et le phénotype a été apporté par la réversion du phénotype grâce à l'introduction par transgenèse d'un fragment de $13,5 \mathrm{~kb}$ provenant du gène d'une souris normale [9]. Ces animaux transgéniques avaient récupéré une expression normale de la GnRH dans I'hypothalamus et un développement normal des organes reproducteurs leur permettant de se reproduire.

\section{Une mutation du gène GNRHI}

dans les hypogonadismes

hypogonadotrophiques chez l'homme

Chez l'homme, jusqu'à très récemment et malgré les efforts de plusieurs équipes à travers le monde, aucune preuve de l'implication du gène GNRHI dans les hypogonadismes hypogonadotrophiques congénitaux n'avait été apportée. Aucune des études réalisées en utilisant différentes approches (Southern blot ou des techniques plus modernes cherchant des délétions, séquençage direct de la partie codante, analyse de liaison génétique) n'avait apporté d'arguments en faveur 


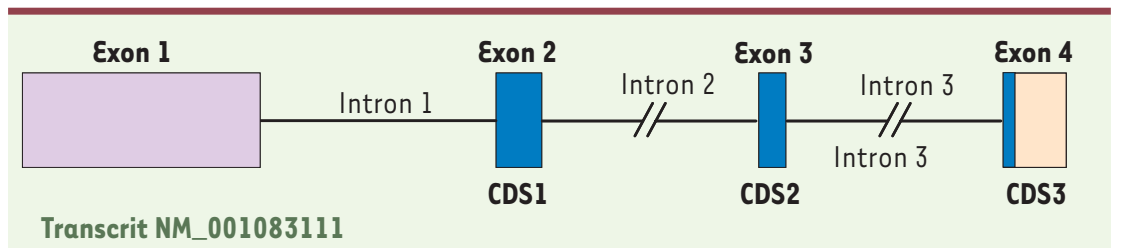

de l'implication de ce gène dans des formes sporadiques ou même familiales d'hypogonadisme hypogonadotrophique congénital, indiquant que les anomalies de ce gène sont un événement germinal extrêmement rare ou bien qu'elles sont non viables chez l'homme. Seuls des polymorphismes avaient été identifiés sans que l'on puisse établir une relation causale solide avec la maladie. Début 2008, nous avons enfin identifié une anomalie génétique de GNRHI dans un cas d'hypogonadisme hypogonadotrophique congénital hypothalamique familial touchant des apparentés des deux sexes en accord avec la localisation autosomique de ce gène [10]. II s'agissait d'une insertion homozygote d'une adénine (c.1819insA) entraînant un décalage du cadre de lecture à partir de l'extrémité aminoterminale au niveau du peptide signal qui était tronqué (Figure 2). Cette anomalie entraînait aussi et surtout une délétion homozygote de la totalité de la séquence du décapeptide. La délétion prévisible de la GnRH induite par cette mutation a été démontrée in vitro dans une lignée expri- mant les enzymes proconvertases, nécessaires à la maturation du préproGnRH. À l'état hétérozygote l'anomalie n'avait pas de conséquence phénotypique apparente excluant une haploinsuffisance. Chez la femme atteinte, le déficit en gonadotrophines a été corrigé par l'administration pulsatile de GnRH exogène ce qui montre l'existence, comme chez la souris hpg, d'une atteinte hypothalamique. Le GnRH exogène rétablissait en effet LH hypophysaire et une sécrétion de FSH permettant une stimulation ovarienne avec une croissance folliculaire et la mise en place des sécrétions stéroïdiennes et peptidiques physiologiques. Ce travail valide donc de façon formelle l'implication de ce gène dans le contrôle hypothalamique de l'axe gonadotrope et écarte complètement l'existence d'une redondance entre la $\mathrm{GnRH}$ l et la $\mathrm{GnRH} 2$ qui est aussi exprimée dans le système nerveux central [5].

${ }^{1}$ Les neuro-hormones sont libérées de façon pulsatile pendant le nycthémère. une sécrétion hypophysaire pulsatile ${ }^{1}$ de
Figure 1. Transcrit de GNRH1 exprimé au niveau hypothalamique. Ce variant contient 4 exons (rectangles). Les introns sont indiqués par des traits et les régions codant pour le préproGnRH (CDS) sont en bleu.

De façon intéressante, les sujets porteurs de la mutation homozygote du GNRHI n'avaient aucune élévation de la prolactine, ce qui excluait que le prolactin inhibiting factor (PIF) soit codé par ce gène contrairement à ce qu'avait affirmé il y a plus de 30 ans le groupe de Seeburg [11]. Qui plus est, la prolactine des malades était plutôt basse, comme chez les souris hpg, probablement en rapport avec la carence œstrogénique. Depuis cette publication princeps, un groupe de Harvard a rapporté un nouveau cas de mutation homozygote chez un garçon de 13 ans ayant un micropénis et une cryptorchidie [12]. Cette nouvelle mutation entraîne un décalage du cadre de lecture qui altère partiellement la séquence du décapeptide. Malgré l'absence de démonstration fonctionnelle dans cet article, on peut anticiper et faire l'hypothèse que, vu le caractère très conservé de la séquence peptidique de la $\mathrm{GnRH}$, cette mutation est probablement délétère et explique le phénotype observé. $\diamond$ GNRH 1 mutation in familial hypogonadotropic hypogonadism

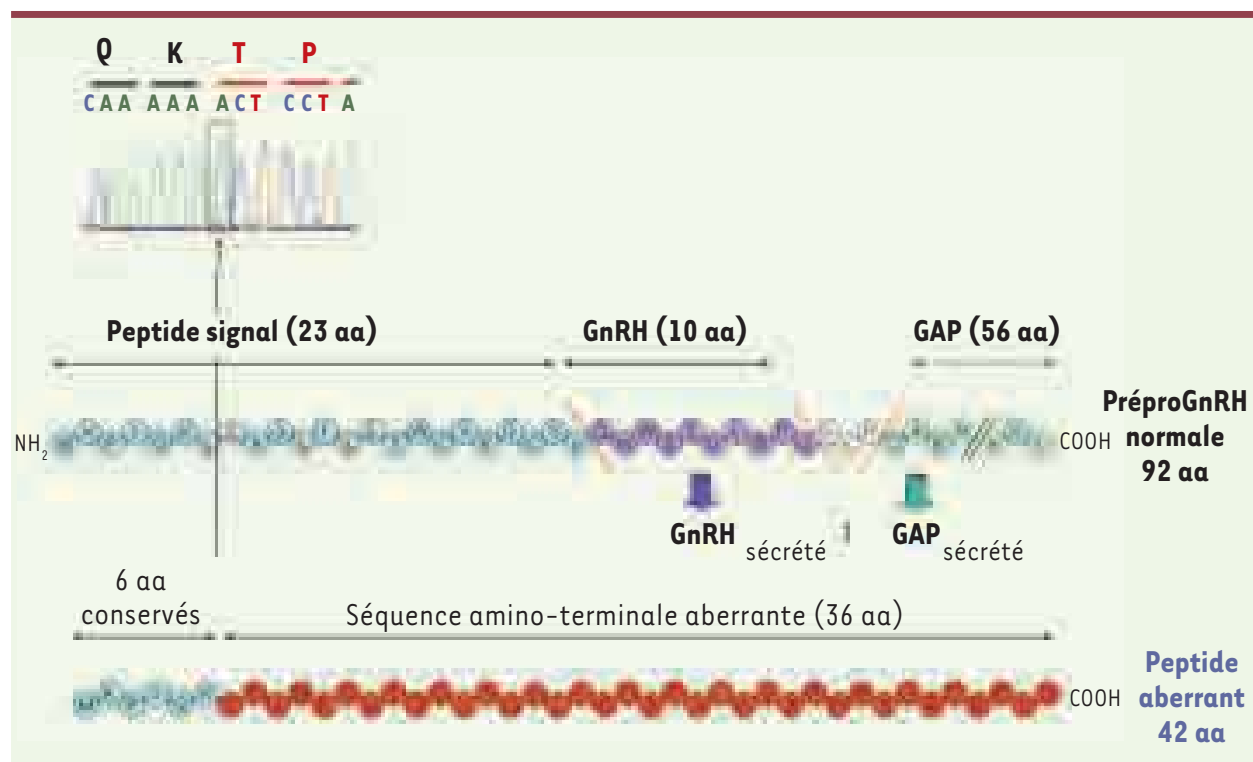

Figure 2. Mutation homozygote c.18-19 ins A du GNRHI chez l'homme. Cette mutation a été mise en évidence chez un adolescent de 18 ans et sa sœur de 17 ans n'ayant aucun développement pubertaire. L'exploration a montré que tous les deux étaient atteints d'hypogonadisme hypogonadotrophique congénital avec olfaction normale. Les conséquences de la mutation sur la séquence peptidique de la préproGnRH sont indiquées (adapté de [10]). 


\section{CONFLIT D'INTÉRÊTS}

Les auteurs déclarent n'avoir aucun conflit d'intérêts concernant les données publiées dans cet article.

\section{RÉFÉRENCES}

1. Fulton JF, Ranson SW, Frantz AM. The hypothalamus and control levels of autonomic functions. Baltimore, MD : Williams and Co, 1940.

2. Guillemin R. Hypothalamic hormones a.k.a. hypothalamic releasing factors. $J$ Endocrinol 2005 184: 11-28.

3. Cattanach BM, Iddon CA, Charlton HM, et al. Gonadotrophin-releasing hormone deficiency in a mutant mouse with hypogonadism. Nature 1977 ; $269: 338-40$.

4. Adelman JP, Mason AJ, Hayflick JS, et al. Isolation of the gene and hypothalamic cDNA for the common precursor of gonadotropin-releasing hormone and prolactin release-inhibiting factor in human and rat. Proc Natl Acad Sci USA 1986; 83 : 179-83.

5. Cheng CK, Leung PC. Molecular biology of gonadotropinreleasing hormone (GnRH)-I, GnRH-II, and their receptors in humans. Endocr Rev 2005 ; $26: 283-306$.

6. Mason AJ, Hayflick JS, Zoeller RT, et al. A deletion truncating the gonadotropin-releasing hormone gene is responsible for hypogonadism in the hpg mouse. Science $1986 ; 234$ : 1366-71.

7. Charlton HM, Halpin DM, Iddon C, et al. The effects of daily administration of single and multiple injections of gonadotropin-releasing hormone on pituitary and gonadal function in the hypogonadal (hpg) mouse. Endocrinology $1983 ; 113$ : 535-44.

8. Gill JC, Wadas $B$, Chen $P$, et al. The gonadotropinreleasing hormone $(\mathrm{GnRH})$ neuronal population is normal in size and distribution in $\mathrm{GnRH}$-deficient and GnRH receptor-mutant hypogonadal mice. Endocrinology 2008 ; 149 : 4596-604.

9. Mason AJ, Pitts SL, Nikolics K, et al. The hypogonadal mouse: reproductive functions restored by gene therapy. Science $1986 ; 234$ : 1372-8.

10. Bouligand J, Ghervan C, Tello JA, et al. Isolated familial hypogonadotropic hypogonadism and a GNRHl mutation. N Engl J Med 2009; 360 : 2742-8.

11. Nikolics K, Mason AJ, Szönyi $\varepsilon$, et al. A prolactininhibiting factor within the precursor for human gonadotropin-releasing hormone. Nature 1985 ; 316: $511-7$.

12. Chan YM, de Guillebon A, Lang-Muritano M, et al. GNRHl mutations in patients with idiopathic hypogonadotropic hypogonadism. Proc Natl Acad Sci USA 2009; 106 : 11703-8.

\section{NOUVELLE}

\section{P53, ARF et P16 : la ligne Maginot de la reprogrammation cellulaire}

Marilyne Dijon-Grinand, John De Vos
Institut de recherche en biothérapie, IRB, Inserm U847, Équipe développement embryonnaire précoce et cellules souches, Hôpital Saint-દloi, 80 , avenue A. Fliche, 34295 Montpellier France. john.devos@inserm.fre-mail
> La technologie des cellules souches pluripotentes induites (iPS), une technique de reprogrammation cellulaire, vient de fêter ses trois ans au mois d'août [1]. On a de la peine à imaginer que cette approche qui a révolutionné le domaine des cellules souches soit si jeune! La recette est simple: l'expression forcée des gènes 0ct4, Sox2, Klf4 et cMyc par manipulation génétique ou par protéines recombinantes contraint une cellule différenciée tel un fibroblaste ou une cellule épithéliale à changer sa nature et à adopter un comportement de type cellule souche embryonnaire [2, 3]. C'est une technique parfaitement reproductible, ce qui explique sa diffusion dans les laboratoires. Cependant, on s'étonne que le pourcentage de cellules reprogrammées à chaque expérience soit si faible, de l'ordre de 0,05-0,5\% pour des fibroblastes embryonnaires de souris ou de 0,002-0,02\% pour des fibroblastes post-natals humains. Cette faible fréquence ne peut s'expliquer par la sélection de rares événements de muta- genèse insertionnelle puisque l'on peut obtenir des iPS par vecteur épisomique ou par protéines recombinantes [3, 4], ni par le ciblage d'une sous-population mineure de cellules souches immatures, puisque la reprogrammation de lymphocytes $B$ matures ayant recombiné le locus des immunoglobulines est possible [5]. Une série exceptionnelle de cinq articles publiés dans la revue Nature vient montrer qu'un frein majeur à la reprogrammation, qui explique en partie le faible pourcentage de cellules reprogrammées, est la voie anti-oncogénique faisant intervenir P53 et INK4a [6-10]. Le premier message de ces travaux est que le blocage de l'activité de P53 augmente considérablement le rendement de la reprogrammation: celui-ci atteint $80 \%$ dans le contexte de la reprogrammation de fibroblastes murins embryonnaires (MEF) « secondaires » (c'est-à-dire issus eux-mêmes d'iPS primaires) par induction par la doxycycline des transgènes intégrés. Ces résultats sont transposables aux cellules humaines et l'efficacité de la reprogrammation de fibroblastes est augmentée d'un facteur 10 [10]. Une telle amélioration de la technologie de reprogrammation ouvre naturellement de nouvelles portes, et, par exemple, le blocage de P53 permet de reprogrammer un certain nombre de types cellulaires réfractaires à la méthodologie «standard » comme les lymphocytes $T$ [7] ou les fibroblastes issus de souris déficientes en télomèrase $\mathrm{hTert}^{-/-}$de troisième génération [10]. Il permet également de raccourcir de manière spectaculaire le temps pendant lequel la surexpression des facteurs de reprogrammation exogènes est requise, passant de 8 jours à 3 ou 4 jours pour des MEF [9], ou de reprogrammer sans vecteur intégratif des cellules jusqu'à maintenant résistantes à ce type de reprogrammation plus délicate [7]. Le blocage de la voie P53 ne doit pas être permanent: son inhibition transitoire par l'utilisation de shARN (short hairpin) ciblant P53 pendant la reprogrammation suffit tout comme son inhibition indirecte par l'intermédiaire de 